\title{
Le pavillon et l'immeuble : géopolitique de la densification dans la région métropolitaine de Lyon ${ }^{1}$
}

\author{
Éric Charmes ${ }^{\mathrm{a}}$ et Max Rousseau ${ }^{\mathrm{b} *}$ \\ ${ }^{a}$ ENTPE (Université de Lyon), RIVES (UMR CNRS EVS), \\ Rue Maurice Audin, 69518 Vaulx-en-Velin Cedex \\ ${ }^{b}$ CIRAD, UMR Art-Dev, Rue Jean-François Breton \\ bâtiment 15, 34398 Montpellier Cedex 5
}

\section{Résumé}

Au nom du développement durable, il faudrait rendre les villes compactes, c'est-à-dire notamment les densifier et lutter contre l'étalement urbain. Cet impératif est souvent présenté sous un angle purement technique. L'objectif de cet article est de mettre en évidence les enjeux politiques et sociaux des politiques visant la compacité des villes. Nous proposons d'analyser cette question en termes de géopolitique métropolitaine. La diversité des territoires métropolitains n'est en effet pas seulement morphologique : elle est aussi économique, sociale et politique. Ces questions sont abordées à partir du cas de la région métropolitaine de Lyon. Comment les territoires très variés qui la constituent réceptionnent-ils l'évolution de la législation nationale concernant la lutte contre l'étalement urbain et la promotion de la «ville compacte »? Comment, au sein d'une région métropolitaine, se négocie le partage d'une densification perçue comme un fardeau ou comme une ressource selon les types de territoires? Finalement, comment les compromis politiques entre territoires se recomposent-ils? La première partie de cet article est ainsi consacrée à

\footnotetext{
${ }^{1}$ Cette recherche a en partie été financée par l'Agence nationale de la recherche dans le cadre du programme "Villes durables " lancé en 2009 et par le Plan urbanisme construction architecture (PUCA). Nous remercions par ailleurs Olivier Frérot, Sébastien Rolland et les deux relecteurs anonymes de Géographie, Économie, Société pour leurs commentaires précieux et pour leurs propositions d'amélioration. Bien évidemment, les erreurs qui demeurent sont les nôtres.
}

*Auteur correspondant : max.rousseau@ cirad.fr 
l'analyse du face-à-face entre les cœurs métropolitains et les couronnes périurbaines. La seconde porte quant à elle sur les négociations internes au cœur métropolitain de politiques de densification en partie contraintes par l'institutionnalisation progressive du périurbain.

Mots clés : politiques de compacité, géopolitique métropolitaine, politiques urbaines, gouvernance.

(C) 2014 Lavoisier, Paris. Tous droits réservés.

\section{Summary}

The suburban house and the apartment block: a geopolitics of urban consolidation in the city-region of Lyon. In the name of sustainable development, cities should be made compact, that is to say denser and less prone to urban sprawl. This requirement is often presented under a purely technical aspect. The objective of this article is to highlight the political and social stakes of the policies aiming at making cities more compact. We propose to analyze this question in terms of metropolitan geopolitics. The diversity of metropolitan areas is indeed not only morphological: it is also economic, social and political. These issues are discussed in the case of the city-region of Lyon. How do the highly diversified territories that constitute this extended metropolis implement the national legislation on urban sprawl and the «compact city»? Within a city-region, how is negotiated the share of an urban consolidation which is perceived either as a burden or as a resource according to the different types of territories? Finally, how are the political compromises between the territories rebuilt at the scale of the extended metropolis? The first part of this article is devoted to the analysis of face-to-face relations between the metropolitan core and the peri-urban areas. The second part deals with the «sub-metropolitan» negotiation of urban consolidation policies, partly constrained by the progressive institutionalization of peri-urban territories.

Keywords: densification policies, metropolitan geopolitics, urban policies, governance.

() 2014 Lavoisier, Paris. Tous droits réservés.

\section{Introduction}

La recherche urbaine critique montre que la montée en puissance récente des discours consensuels sur le développement durable s'accompagne d'une dépolitisation des enjeux (Reigner, 2013; Béal et al., 2011; Pinson et Rousseau, 2011). De ce point de vue, le poids pris par l'idéologie du développement durable peut s'analyser comme l'une des manifestations d'une transition de longue durée que Colin Crouch (2005) perçoit comme celle conduisant de la démocratie, caractérisée par la prégnance de grands conflits idéologiques émanant de conflits de classe, vers la société «post-démocratique » dans laquelle les débats sur les enjeux politiques, sociaux et économiques s'effacent au profit de discussions de plus en plus techniques, circonscrites au cadre restreint des élites politiques et économiques.

Les politiques de compacité urbaine sont très marquées par cette dépolitisation. Au nom du développement durable, s'affirme comme indiscutable la nécessité de rendre les villes compactes, c'est-à-dire entre autres choses de les densifier et de lutter contre l'étalement urbain (OCDE, 2012). Ces deux derniers impératifs sont complémentaires : en contenant les extensions périphériques, on favorise la poussée urbaine au centre, et 
réciproquement. On pourrait discuter des bénéfices environnementaux de la compacité (Nessi, 2012; Echenique, 2012). Mais ce n'est pas ici notre propos. L'objectif de cet article est de mettre en évidence les enjeux politiques et sociaux de certaines des politiques mises en œuvre pour assurer la durabilité des villes. En réalité, la compacité peut être définie de manière très variable et les politiques locales qu'elle inspire sont ellesmêmes très diverses (Touati, 2013; Charmes, Keil, à paraître). En outre, les politiques et prescriptions portées par l'État sont souvent en tension. Comme on le verra par exemple, les politiques d'aide à l'accession à la propriété des ménages modestes contrecarrent largement la lutte contre l'étalement urbain. Nous proposons d'analyser ces questions en termes de géopolitique de l'aménagement du territoire (Subra, 2007), en montrant comment à l'échelle d'une région métropolitaine ${ }^{2}$ importante se nouent des coalitions ou des associations défendant l'intérêt de territoires locaux aux caractéristiques différentes. Cette approche s'avère selon nous particulièrement adaptée pour comprendre les difficultés auxquelles se heurte en France le gouvernement des régions métropolitaines.

Les enjeux de la lutte contre l'étalement et de la densification sont ici abordés à partir du cas de la région métropolitaine de Lyon. Comment les territoires très variés qui constituent cette métropole élargie réceptionnent-ils l'évolution de la législation nationale concernant la lutte de l'étalement urbain et la promotion de la densité ? Comment, au sein d'une aire métropolitaine, se négocie le partage d'une densification perçue comme un fardeau ou comme une ressource selon les types de territoires? Finalement, comment les compromis politiques entre territoires se recomposent-ils? La diversité des territoires métropolitains n'est en effet pas seulement morphologique, elle est aussi économique, sociale et politique. Ainsi, le peuplement des quartiers les plus réceptifs à l'exigence de densification diffère de celui des quartiers qui y résistent. C'est une évidence, mais cette évidence est souvent absente des débats sur la nécessité de densifier la ville.

Au-delà de cette mise en évidence des enjeux géopolitiques des transformations menées dans les métropoles au nom du développement durable, cet article contribue à la réflexion sur le gouvernement des grandes villes par l'échelle qu'il embrasse. Le territoire métropolitain est en effet appréhendé ici en incluant les couronnes périurbaines. Même s'il occupe une place croissante dans les débats académiques et dans les politiques publiques, le périurbain reste encore peu considéré. Il est aussi mal compris. Dans la littérature de science politique sur les villes, hormis quelques exceptions, les couronnes périurbaines soit sont absentes, soit apparaissent comme le prolongement des banlieues. L'idée que le périurbain pourrait être un espace durablement doté d'une spécificité reste peu présente. Dans cet article, nous partirons de cette hypothèse, en nous appuyant sur nos propres travaux (Charmes, 2011) et sur ceux de Philippe Estèbe (2008) et Martin Vanier (2008). Par ailleurs, au cours des dernières années, les espaces périurbains ont commencé à surmonter leur diversité afin de présenter un intérêt commun opposable à l'échelle verticale - l'État -, mais aussi horizontale - les cœurs métropolitains, avec lesquels les espaces périurbains nouent une relation ambivalente, entre dépendance et concurrence. Ainsi en utilisant la double incitation à la densification et à la lutte contre

\footnotetext{
${ }^{2}$ Dans cet article, on désignera par cœur métropolitain la partie centrale de la région métropolitaine (constituée, dans le cas de Lyon, par la communauté urbaine); par aire urbaine, l'ensemble constitué par un grand pôle urbain et sa couronne périurbaine au sens de l'INSEE; et par région métropolitaine, un vaste ensemble incluant l'aire urbaine.
} 
l'étalement urbain en provenance de l'État comme outil d'analyse, la première partie de cet article est consacrée à l'analyse du face-à-face entre le cœur métropolitain de Lyon et ses couronnes périurbaines. La seconde partie porte quant à elle sur les négociations internes au cour métropolitain pour les politiques de densification, négociations en partie contraintes par l'institutionnalisation progressive du périurbain.

\section{Encadré 1 : L'aire urbaine de Lyon, un territoire contrasté doublé d'une géopolitique complexe}

D'après le zonage de 2010 de l'INSEE, l'aire urbaine de Lyon compte 514 communes et 2,1 millions d'habitants (en 2008). Dans cet ensemble, 384 communes sont périurbaines et 130 composent le pôle urbain, c'est-à-dire l'agglomération centrale. 58 des communes du pôle urbain et 1,3 million d'habitants sont rassemblés dans une communauté urbaine, celle du Grand Lyon, dont le centre est constitué des villes de Lyon et Villeurbanne. Marqué par une ségrégation historique entre la banlieue Ouest, résidentielle, aisée et pavillonnaire, et la banlieue Est, industrielle, populaire et constellée de grands ensembles, le pôle urbain de Lyon est constitué d'un ensemble de territoires fortement disparates, aux caractéristiques morphologiques, économiques, sociales et politiques hétérogènes, mais qui présentent pour point commun de subir depuis une décennie une incitation croissante à la densification en provenance de l'État central ainsi que du Grand Lyon.

Les couronnes périurbaines de Lyon sont également très diverses (Davezies, Estèbe, 2007). La division Est/Ouest se prolonge, avec à l'Est des territoires dominés par des plaines à la fois résidentielles et industrielles, notamment dans le Bugey, et à l'Ouest des territoires dont le relief beaucoup plus marqué a limité une croissance urbaine dominée par les ensembles pavillonnaires et marquée par l'accueil de populations plus favorisées qu'à l'Est (sachant toutefois que l'opposition sociale entre l'Ouest et l'Est se révèle, à l'échelle du périurbain lyonnais, beaucoup moins marquée qu'à l'échelle des banlieues et que, d'une manière générale, le spectre social est beaucoup plus large dans le cœur métropolitain que dans le périurbain, où les positions extrêmes sont moins représentées). Cette division en quadrants est doublée d'une division en fonction de la distance au cœur de la métropole, les territoires concentrant des populations de plus en plus modestes au fil de l'augmentation de cette distance. À l'Ouest, dans les Monts du Lyonnais, et au Nord, dans le Beaujolais, les populations deviennent aussi plus âgées.

La diversité sociale et économique caractérisant l'aire urbaine de Lyon se double d'une fragmentation politique. Si le cœur métropolitain fait l'objet d'une lente intégration sous l'égide de la communauté urbaine de Lyon, les échanges entre les communes et la structure intercommunale reposent toujours sur un jeu politique complexe, comme nous le verrons dans cet article. À l'échelle de l'aire urbaine et de la région, la fragmentation politique est encore plus importante puisque des dizaines de communautés de communes et pas moins de treize syndicats mixtes porteurs de schémas de cohérence territoriale se partagent le territoire. Ces syndicats défendent les intérêts de territoires porteurs de caractéristiques contrastées, ce qui explique la difficulté de coordonner leur action - bien qu'une démarche de coopération inter-Scot ait été mise en place dès 2003 par l'agence d'urbanisme de Lyon (voir Encadré 2). 


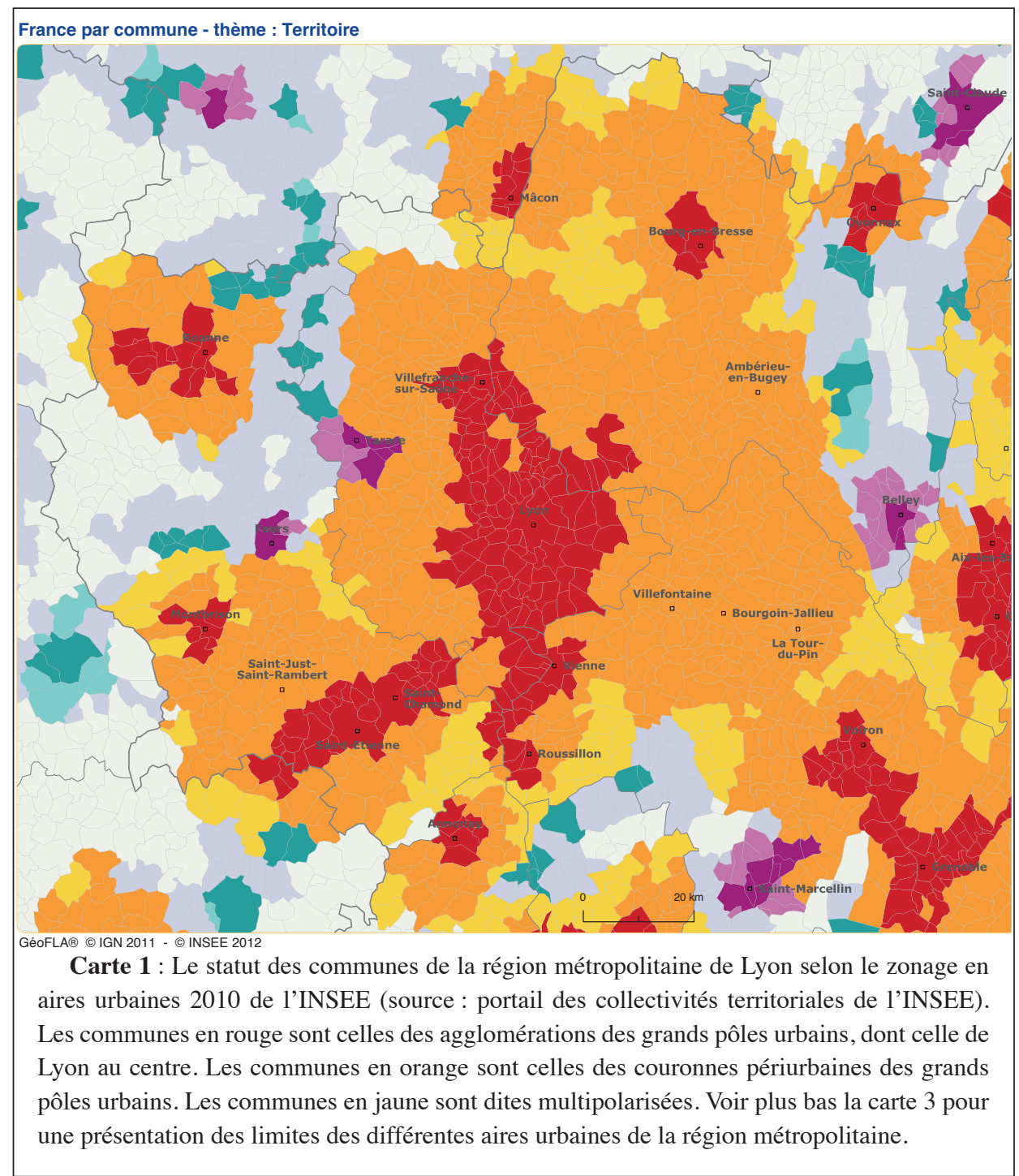

Cet article repose sur la réalisation d'une quarantaine d'entretiens. Dans le territoire du Grand Lyon, une vingtaine d'entretiens semi-directifs ont été effectués en 2011 avec des élus et des responsables administratifs de communes de la première couronne de l'agglomération lyonnaise. Dans le périurbain, des enquêtes ont été menées en 2006 auprès d'une douzaine d'élus du territoire couvert par le schéma de cohérence territoriale (SCOT) de l'Ouest lyonnais. Une enquête a aussi été réalisée en 2012 par Hélène Hicaubé, dans le cadre d'un travail de fin d'études, sur les SCOT des territoires périurbains de la région métropolitaine de Lyon (Hicaubé, 2012). Enfin, l'enquête a été complétée par des entretiens menés auprès d'élus et de responsables administratifs du Grand Lyon et de l'agence d'urbanisme de Lyon. 


\section{Il court, il court, l'étalement : la métropole face à ses périphéries}

On pourrait a priori s'attendre à ce que les communes périurbaines s'opposent aux politiques de lutte contre l'étalement qui invitent nombre d'entre elles à limiter leur croissance démographique, au profit de quelques communes bien desservies par les transports collectifs. Comme on va le voir, la réalité est plus complexe. Les territoires périurbains sont divers et leurs intérêts le sont tout autant. Certains ne souhaitent rien tant que limiter leur croissance, voire la stopper. C'est ainsi qu'une convergence d'intérêts a pu s'établir entre un Grand Lyon désireux de concentrer la croissance urbaine sur son territoire et des premières couronnes plus adeptes du malthusianisme. Mais cette convergence d'intérêts est en trompe-l'œil car elle ne concerne pas tous les territoires périurbains, et parce que la périurbanisation est un phénomène dynamique. Ainsi, loin de limiter l'étalement urbain, elle l'accentue.

Par ailleurs, l'enjeu de cet étalement urbain n'est pas uniquement environnemental. Les questions posées ne sont pas seulement celles de l'artificialisation des sols, de la consommation d'énergie ou des émissions de gaz à effet de serre. L'étalement urbain est associé à un éloignement des accédants modestes à la propriété, éloignement qui pèse lourdement sur les budgets des ménages et soulève des questions de justice sociale, notamment du point de vue du droit aux transports. Avec l'étalement se trouve également questionnée la capacité de la métropole lyonnaise à maintenir en son cœur ou à proximité les familles des classes moyennes inférieures. L'enjeu est en partie fiscal. Il est aussi économique : comme l'a rappelé notamment Ludovic Halbert (2010), les cadres supérieurs ne sont pas les seuls moteurs de l'économie métropolitaine, les employés, ouvriers et professions intermédiaires apportent également une contribution essentielle.

Cette première partie propose donc une lecture géopolitique des politiques de compacité urbaine à l'échelle de l'aire urbaine et plus largement de la région métropolitaine. Il s'agit d'analyser les rapports entre le cœur métropolitain, dont l'intérêt est représenté par la communauté urbaine de Lyon, et les territoires périurbains (ou qui pourraient le devenir), dont les intérêts sont notamment exprimés dans des schémas de cohérence territoriale.

\subsection{Quand les territoires périurbains acquiescent à la lutte contre l'étalement urbain}

Au sein du Grand Lyon, les mises en cause successives du modèle de suburbanisation «post-fordiste » des métropoles françaises, basé sur l'initiative du secteur privé, l'habitat individuel et l'étalement urbain, entrent en écho avec les préoccupations croissantes de la communauté urbaine pour la compétitivité économique de la métropole à l'échelle globale. À partir du début des années 1990, sous l'influence d'une nouvelle génération d'élus, la communauté urbaine de Lyon s'engage en effet clairement dans la voie du développement économique entrepreneurial en conduisant une stratégie axée sur l'attraction des investissements extérieurs et la tertiarisation de l'économie locale (Jouve, 2001). Toutefois, ce nouveau discours sur la compétitivité mondiale de l'agglomération lyonnaise paraît se heurter au poids démographique croissant des périphéries situées hors du périmètre d'influence de la communauté urbaine. Constituée de petites communes périurbaines, cette périphérie connaît une forte expansion sur la base de l'éta- 
lement urbain. En outre, l'institutionnalisation politique accélérée des territoires périurbains (voir Encadré 2) favorise la cristallisation d'un modèle de développement urbain en confrontation directe avec le cœur métropolitain.

Face à des territoires périurbains défendant un modèle axé sur les faibles densités, et concurrençant le cœur métropolitain pour l'attraction des ressources nécessaires à son développement (flux d'actifs, équipements structurants, activités économiques), le Grand Lyon perçoit rapidement comme une opportunité la nouvelle législation nationale incitant à la densification avec notamment la loi Solidarité et renouvellement urbains (SRU) de 2000.

\section{Encadré 2 : L'institutionnalisation du périurbain, quand les cours des métropoles rencontrent un nouvel interlocuteur}

Face à l'incitation à l'intercommunalité, les élus périurbains se sont généralement montrés réticents devant l'intégration de leur commune dans la communauté urbaine voisine. Ils se sont notamment inquiétés d'une dilution de leur pouvoir au sein d'un conseil communautaire où, pour des raisons démographiques, ils ne pouvaient espérer qu'une influence modeste. Mais ils ont également rapidement compris qu'il était vain de résister au mouvement et qu'il valait mieux prendre les devants pour choisir sa communauté plutôt que de se le faire imposer par un préfet. Ainsi de très nombreux élus se sont tournés vers leurs voisins périurbains pour créer des communautés de communes périurbaines.
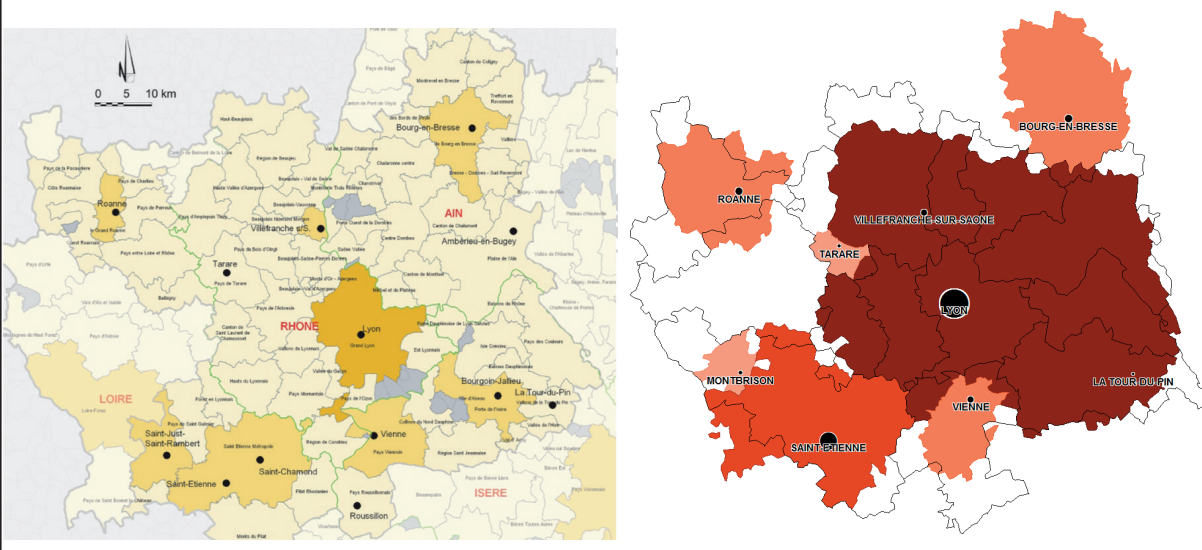

Carte 2 (à gauche) : Les communautés de communes autour du Grand Lyon (en jaune foncé). Les communautés d'agglomération apparaissent en jaune clair et les communes qui n'appartiennent à aucune intercommunalité apparaissent en jaune moyen (source : Région urbaine de Lyon. La carte représente la situation au $1^{\mathrm{er}}$ janvier 2007).

Carte 3 (à droite) : Les limites des SCOT, en traits noirs, et, en aplats de couleur, les aires urbaines de la région métropolitaine lyonnaise (cartographie Hélène Hicaubé, 2012). 
Loin d'être à l'échelle des métropoles, ces communautés de communes périurbaines sont plutôt à l'échelle des cantons, avec en leur centre un bourg ou parfois une petite ville. Elles encerclent les communautés urbaines (ou d'agglomération) et font obstacle à l'extension de ces dernières. Hors des limites de la communauté urbaine de Lyon, les communes sont ainsi réparties dans des dizaines de communautés. Certaines privent le Grand Lyon du contrôle de ressources et d'équipements importants. Ainsi, le Grand Lyon a longtemps eu des velléités d'inclure l'aéroport Saint-Exupéry dans son périmètre. Mais les communes qui avoisinent l'aéroport se sont unies pour faire front et préserver leurs ressources fiscales.

Avec la loi SRU de 2000, le législateur escomptait inciter les communes périurbaines à intégrer les structures chargées d'élaborer les documents de planification des métropoles, les SCOT. Ce n'est cependant pas ce qui s'est produit. Beaucoup de communes périurbaines se sont associées pour mettre en place des SCOT défendant leurs intérêts "périurbains » face aux agglomérations. Ainsi, si certaines métropoles françaises sont parvenues à se doter d'un SCOT qui couvre leur aire urbaine, beaucoup d'autres n'ont pas atteint cet objectif. L'aire urbaine de Lyon est ainsi associée à une dizaine de SCOT. Le projet d'un grand SCOT ne subsiste plus qu'à travers un espace de dialogue appelé Inter-SCOT et piloté par les agences d'urbanisme de Lyon et de Saint-Étienne.

Paradoxalement, à l'échelle de l'aire urbaine, la volonté du Grand Lyon de capter la croissance sur son territoire au travers de la densification est loin de se heurter aux résistances auxquelles on pourrait s'attendre. En réalité, dans les communes périurbaines les plus proches du centre de Lyon, la croissance des espaces bâtis atteint souvent rapidement une sorte de plafond. Comme l'illustre le cliché 1, dans les communes périurbaines, le taux d'urbanisation est très faible et le paysage est souvent dominé par les espaces naturels et agricoles. D'après des estimations de Jean Cavailhès (2004), en France, les couronnes périurbaines ne sont urbanisées qu'à $20 \%$, ce qui signifie que $80 \%$ des sols sont agricoles, forestiers ou naturels. En réalité, en s'installant dans une commune périurbaine, les ménages viennent chercher non seulement un logement (généralement une maison individuelle), mais aussi un cadre de vie campagnard (Charmes, 2005). Un élément central de ce cadre de vie est d'une part l'abondance d'espaces non bâtis naturels ou agricoles, d'autre part la séparation physique d'avec l'agglomération de la métropole voisine. D'où une propension des communes périurbaines à conserver tels quels les espaces non bâtis qui entourent leurs noyaux d'urbanisation. Cette propension est d'autant plus forte qu'elle s'accorde parfaitement avec des politiques de maintien, voire d'élévation du standing des communes. En limitant la croissance, on restreint l'offre et on fait monter les prix, ce qui assure un véritable tri social. 


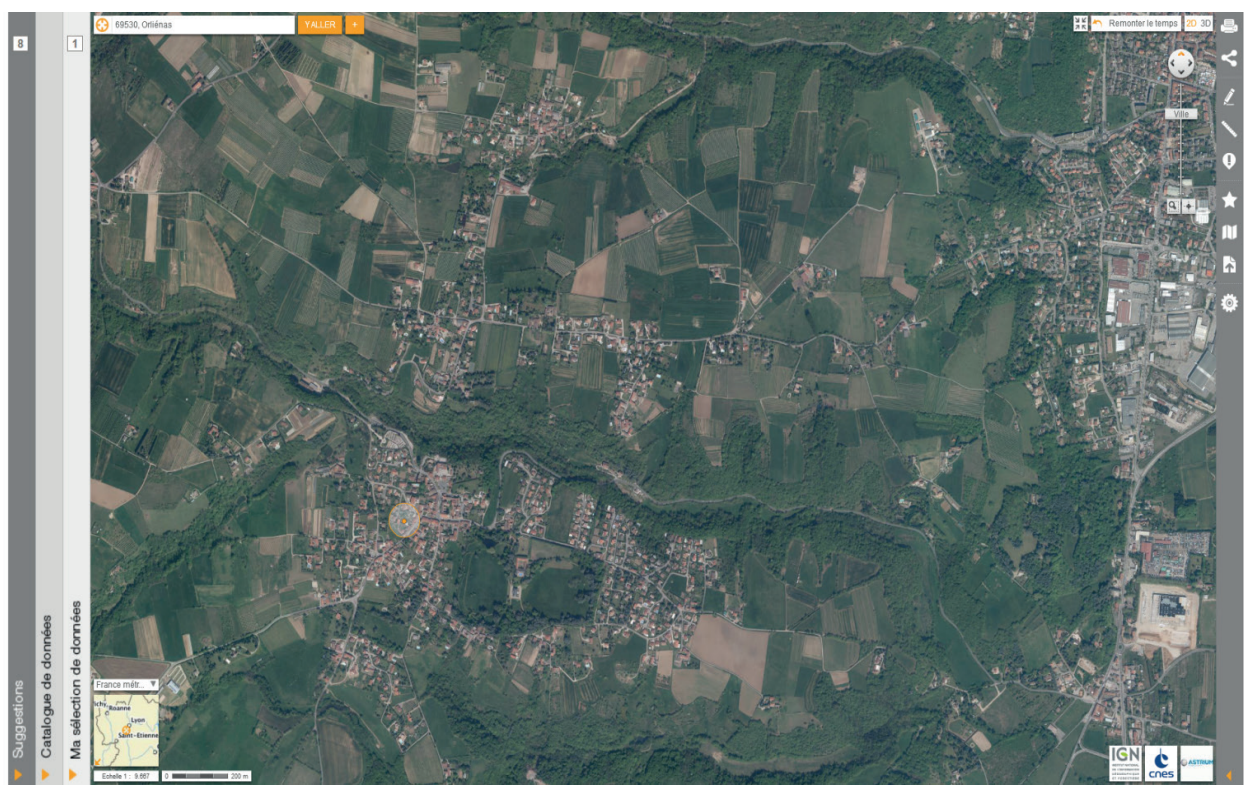

Cliché 1 : La commune d'Orliénas en limite ouest de l'agglomération lyonnaise, qui apparaît à l'est. Cette commune compte un peu moins de 2200 habitants (source : Géoportail, Cliché 2012).

Ainsi, autour de Lyon comme autour des autres grandes métropoles, dans les premières couronnes périurbaines (celles qui se situent à moins d'une quinzaine de kilomètres des limites de l'agglomération principale), la tendance est au malthusianisme foncier, notamment dans les petites communes résidentielles, très nombreuses puisque $85 \%$ des communes de la couronne périurbaine comptent moins de 2000 habitants. Lorsque ces communes élaborent leur plan d'urbanisme, la première préoccupation des habitants est la limitation de la croissance urbaine afin de préserver un cadre villageois. Cette attente est relayée à l'échelle des SCOT. Les SCOT périurbains sont très divers (Davezies, Estèbe, 2007; Hicaubé, 2012) et la place nous manque pour entrer dans les détails, mais leur contenu fait souvent valoir une volonté de chaque commune de ne pas rejoindre la banlieue et la nappe bâtie continue de l'agglomération de Lyon. Ceci se traduit notamment par des mesures de contrôle de l'usage des sols visant à préserver les espaces naturels et agricoles qui entourent chacune de ces communes.

Notre communauté s'est unie à trois autres intercommunalités pour porter, à travers un SCOT un projet de "réseau de villages » [...] L'objectif est de conserver une idée de villages ou de gros bourgs (parce que S. n'est plus vraiment un village, disons un gros bourg), mais qui visuellement ont une identité à la fois socio-culturelle et une identité visuelle. Nous à S. on a la chance, malgré cette pression [foncière] d'avoir des coupures vertes naturelles, puisque la particularité de S. c'est que d'où qu'on vienne, pour rentrer il faut toujours franchir un "pont ». On a quand même une belle couronne verte (entretien, maire d'une commune périurbaine de l'Ouest lyonnais, mars 2006).

Dans ce contexte, les projets périurbains et les projets métropolitains peuvent assez facilement s'accorder. Même si chacun a élaboré ses projets de manière relativement autonome, une convergence d'intérêts émerge entre des espaces périurbains qui limitent leur croissance 
et un centre d'agglomération qui, à l'inverse, recherche la croissance. Ainsi, au sein de l'InterSCOT de la métropole lyonnaise, le dialogue est relativement serein entre les espaces périurbains et le cœur de la métropole. La volonté d'attirer de nouvelles populations semble se concilier harmonieusement avec celle de préserver les espaces naturels et agricoles :

Pour les SCOT, la volonté d'attirer de nouvelles populations s'accompagne d'une volonté d'accorder une place aussi importante aux espaces naturels et agricoles qu'aux espaces urbains, considérant qu'ils représentent une richesse essentielle, d'abord au plan économique à travers l'activité agricole, ensuite au plan social à travers leur valeur d'usage (cadre de vie, tourisme, loisirs) pour les habitants de l'aire métropolitaine, enfin au plan environnemental pour la préservation de la biodiversité (extrait du « chapitre commun » signé en 2013 pour tous les présidents des SCOT de la région métropolitaine lyonnaise, p. 13)

On peut d'ailleurs se demander si les engagements pris dans les SCOT périurbains auraient été très différents si le SCOT du Grand Lyon avait couvert l'ensemble de l'aire urbaine. Le Grand Lyon n'est pas seulement séduit par l'idée de disposer de périphéries dotées d'espaces naturels et agricoles préservés, il trouve aussi son compte dans la réduction de la croissance des communes situées hors de son périmètre : cela lui permet de limiter les migrations résidentielles et d'augmenter les chances de réussite de ses opérations de renouvellement urbain, à commencer par la plus connue, «Lyon Confluence » .

Les relations ne sont guère plus tendues avec l'État. Ce dernier n'intervient pas seulement dans le contexte lyonnais au travers de sa production législative, il intervient aussi par le biais d'un document de planification, la directive territoriale d'aménagement et de développement durable (DTA). Dans cette directive, qui couvre une large part de l'aire urbaine lyonnaise ${ }^{3}$, l'État, en conformité avec les lois actuelles, affirme la nécessité de lutter contre l'étalement urbain. Ce faisant, il affiche des objectifs convergents avec ceux du Grand Lyon et des territoires périurbains. Il s'agit en effet de préserver le patrimoine paysager, de maintenir les espaces agricoles ou de réduire l'imperméabilisation des sols. Il s'agit aussi de favoriser la concentration de la croissance urbaine dans le pôle urbain :

L'étalement urbain, non maîtrisé jusqu'à présent, a empêché le développement de stratégies de recomposition de la ville sur elle-même (extraits de la DTA de l'aire métropolitaine lyonnaise, 2006, p. 17).

Certes, beaucoup de communes périurbaines protestent contre les demandes de l'État et les textes de loi contraignants, mais c'est avant tout pour le principe, pour manifester qu'elles souhaitent conserver leur autonomie et ne pas être soumises à des autorités distantes. Au fond, elles se satisfont bien du rôle qui leur échoit de préserver la couronne verte de la métropole. Elles s'approprient d'ailleurs à leur échelle le discours sur la nécessité de lutter contre l'étalement urbain et expliquent que c'est en partie pour cette raison (et également à cause du coût des équipements collectifs) qu'elles ne peuvent plus créer de quartiers pavillonnaires. Orliénas ${ }^{4}$ est un bon exemple de ce type de posture. Cette commune de 2200 habitants située à une quin-

\footnotetext{
${ }^{3}$ La DTA est téléchargeable à l'adresse suivante : www.rhone-alpes.developpement-durable.gouv.fr/IMG/ pdf/03-dta_aml_cle7584cb.pdf

${ }^{4}$ Selon le dernier zonage de l'INSEE, Orliénas est rattachée au pôle urbain de Lyon. L'analyse d'une photographie aérienne indique cependant clairement l'identité périurbaine de la commune et le lien de continuité de la zone bâtie principale d'Orliénas avec le pôle urbain de Lyon est très ténu, à tel point que l'on peut se demander si un reclassement en commune périurbaine ne s'imposerait pas.
} 
zaine de kilomètres au sud-ouest du centre de Lyon a gagné l'essentiel de ses habitants récents au cours des années 1980 et 1990. La commune a ensuite mis en place des règles d'urbanisme très restrictives afin de limiter toute nouvelle construction. Bien que regrettant la relative perte de ses pouvoirs en matière d'urbanisme, un élu se déclare satisfait des nouvelles préconisations concernant la maîtrise de la croissance, et s'inquiète même de ce que les projets de construction de nouvelles infrastructures routières (notamment le contournement autoroutier ouest de Lyon) risquent d'attiser les convoitises des bâtisseurs :

On fait partie du SCOT de l'Ouest Lyonnais approuvé en février 2011. Dans la directive territoriale d'aménagement, on est une zone de prescription particulière : le plateau est considéré comme étant le poumon vert de l'agglomération lyonnaise, avec des taux de croissance faibles, donc l'objectif est de permettre le redéveloppement de la vallée du Gier en évitant que le plateau mornantais ne la phagocyte. On est en phase car on veut maîtriser notre croissance. Mais parfois il y a une incohérence : on nous demande de ne pas grossir, de garder des espaces verts mais on nous impose des infrastructures routières qui vont entraîner une pression foncière autour des sorties. Ce sont plutôt ces incohérences qui nous choquent, pas les objectifs eux-mêmes. En ce qui concerne la maîtrise de la croissance, tous les villages du plateau mornantais souhaitent garder un cachet de village, et ne pas devenir des villes-dortoirs de l'agglomération lyonnaise. On veut garder des activités et de l'animation. Cela passe par la maîtrise du foncier et l'intégration des nouveaux habitants (entretien, janvier 2011).

Le malthusianisme est certes relatif et de nombreuses communes continuent à connaître des taux de croissance démographique de l'ordre du pourcent. Sans entrer dans le détail des logiques à l'œuvre (voir Vilmin, 2006), les constructions nouvelles et les ouvertures à l'urbanisation sont principalement justifiées par la nécessité d'une part de loger les « enfants du pays » (écartés de l'accession à la propriété d'une maison individuelle par des prix devenus trop élevés), d'autre part d'offrir des logements adaptés aux ménages âgés, dont le poids dans la démographie des premières couronnes périurbaines est de plus en plus lourd. Ce vieillissement conduit également à se préoccuper du maintien d'un nombre d'élèves suffisants pour maintenir les classes ouvertes. Pour satisfaire ces besoins tout en préservant leur cadre de vie résidentiel et verdoyant, les communes orientent la production de logement vers le collectif, pour partie sous la forme d'établissements d'accueil de personnes âgées, et pour partie également sous la forme de logements sociaux, y compris dans l'Ouest lyonnais, secteur périurbain le plus aisé de la région métropolitaine (la chose est d'autant plus notable qu'à de rares exceptions près, les communes périurbaines n'ont pas de contrainte légale dans ce domaine). Outre qu'il permet de résoudre le problème de la solvabilité des jeunes ménages, ce compromis permet d'éviter la construction de pavillons en extension. Il répond de surcroît à la nécessité de restructurer et de réhabiliter des noyaux villageois délaissés jusqu'il y a peu, mais devenus l'objet d'un intérêt grandissant, avec l'augmentation de la pression foncière. Ces politiques d'urbanisme sont en outre habilement mises en résonance avec les discours sur la compacité. Plusieurs communes parlent ainsi de politiques de densification de leur noyau villageois.

Ceci étant, la croissance urbaine, quand elle existe, reste très loin de répondre à la demande solvable. Les ménages modestes en particulier ont des difficultés à trouver leur place. Chaque commune reste en effet très soucieuse de préserver son standing social. Ainsi les logements qui sont produits dans les immeubles collectifs sont souvent de petite 
taille pour éviter que des familles n'envisagent de s'y installer trop durablement. Ces logements doivent rester des logements «pied à l'étrier» pour de jeunes ménages qui attendent de pouvoir acquérir une maison individuelle. Cette opportunité risque d'être difficile à trouver dans les premières couronnes périurbaines pour les ménages des classes moyennes et plus encore pour ceux des classes moyennes inférieures.

\subsection{Quand la lutte contre l'étalement urbain éloigne les accédants à la propriété les plus modestes}

En réalité, le compromis qui émerge, sans avoir nécessairement été recherché, entre la métropole et sa couronne périurbaine est en trompe-l'œil. Les espaces périurbains sont divers, et, surtout, la périurbanisation est un phénomène dynamique. Ainsi, en limitant l'urbanisation dans la première couronne périurbaine, on favorise son report. Les jeunes ménages avec enfants à la recherche d'une maison individuelle doivent chercher plus loin leur bonheur (Wiel, 2002; Charmes, 2011). Et ils le trouvent, car au-delà de la couronne périurbaine existent des communes rurales dont les habitants et les élus sont heureux de pouvoir accueillir de nouveaux ménages, ceci tout d'abord dans la perspective de redynamiser leur village et notamment de repeupler l'école. Les propriétaires bénéficient quant à eux de la valorisation de leurs terres. Entre la terre agricole et le terrain à bâtir, le facteur multiplicatif moyen à l'échelle nationale s'élève à 55 (Levesque, Liorit et Pathier, 2011). Autour des grandes métropoles, et notamment à Lyon, il peut atteindre 100 et pour un hectare la somme en jeu peut être de l'ordre du million d'euros. Par ailleurs, et peut-être même surtout, les politiques sectorielles de l'État ne sont pas exemptes de contradiction et l'étalement que les lois d'urbanisme et d'aménagement veulent contenir, est encouragé par des politiques d'aide à l'accession à la propriété qui soutiennent les ménages modestes à la condition qu'ils achètent des logements neufs, ce qu'ils ne peuvent faire qu'en s'éloignant fortement des cœurs des métropoles (Renard, 2006).

L'extension des villes se fait alors par vagues successives, une couronne périurbaine s'ajoutant à une autre couronne plus proche du pôle urbain lorsque celle-ci se ferme à l'urbanisation (Wiel, 2002). Ainsi, à l'Ouest, l'aire d'influence de Lyon s'est étendue dans des communes des Monts du lyonnais jusqu'alors très rurales et relativement difficiles d'accès en raison du relief. Les marges extérieures de l'aire urbaine s'urbanisent d'autant plus facilement qu'elles sont à l'écart du périmètre de la Directive territoriale d'aménagement de l'État et que, d'une manière générale, elles ne sont pas considérées comme des territoires à enjeux par les acteurs métropolitains. Ainsi, en comparant les aires urbaines de Lyon entre 1999 et 2010 (voir Carte 4), on note une très forte extension des couronnes périurbaines, malgré la loi Solidarité et renouvellement urbains et son renforcement par les lois issues du Grenelle de l'Environnement. Entre le zonage de 1999 et celui de $2010^{5}$, la couronne périurbaine de Lyon a gagné près de 200 communes, passant de 194 à 383 communes. Ce phénomène n'est pas seulement lyonnais. À l'échelle nationale, les couronnes des grandes aires urbaines, celles dont le pôle urbain concentre plus de 10000 emplois, se sont étendues de 39,2 \% (Floch et Lévy, 2011).

\footnotetext{
${ }^{5}$ Le zonage de 2010 a été établi sur la base des données dites de 2008, ce qui veut dire que l'extension a été mesurée sur une période de 9 ans et non de 11 ans.
} 


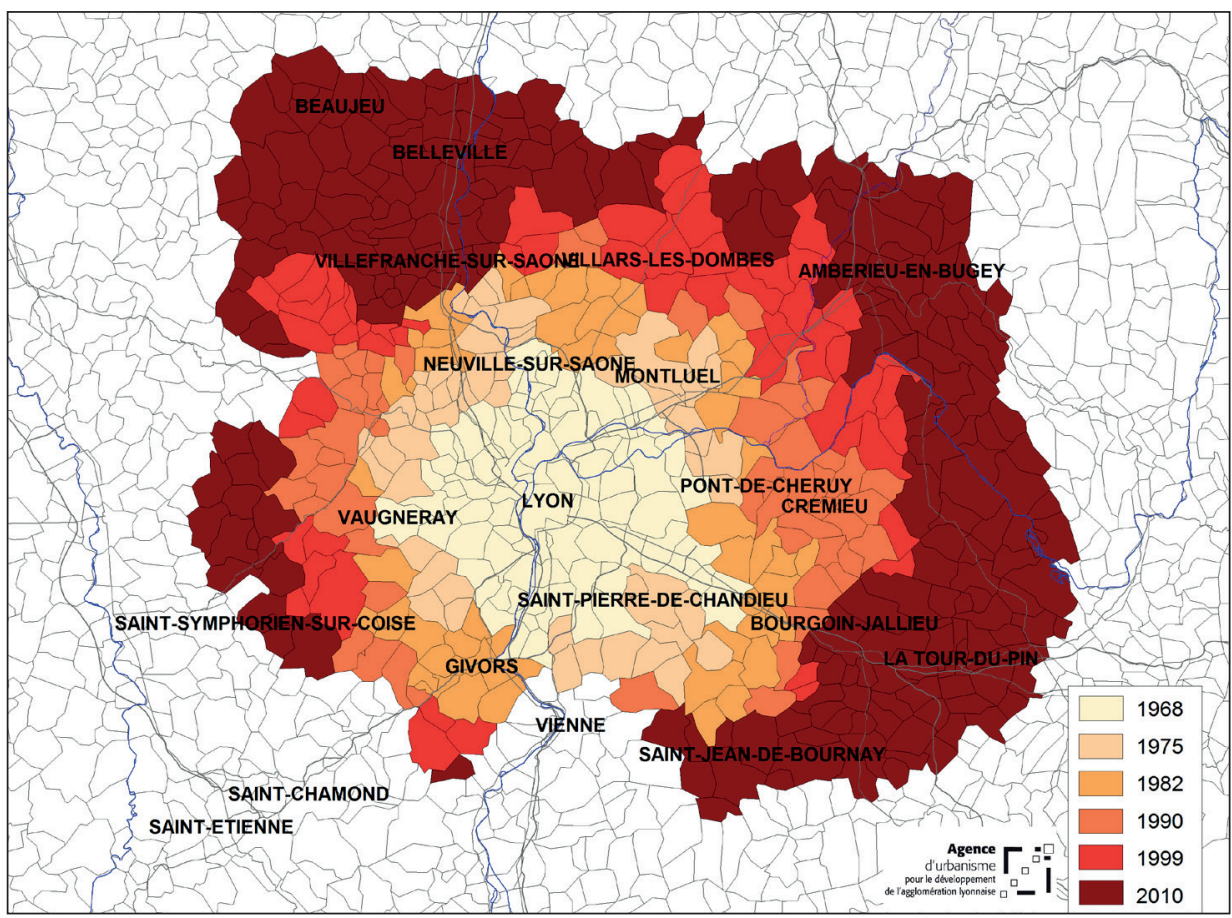

Carte 4 : Extensions de l'aire urbaine de Lyon de 1968 à 2010. Ces extensions sont particulièrement fortes à l'est et à l'ouest dans la période récente (source : Agence d'urbanisme de Lyon, d'après les données de l'INSEE)

On constate dans l'aire urbaine lyonnaise une prise de conscience de cette situation, notamment depuis le tournant des années 2010. La première couronne périurbaine a largement contribué à cette prise de conscience de la vigueur de l'extension des aires urbaines. En effet, cette extension conduit à un accroissement très fort du trafic sur son territoire. Cette couronne n'est plus seulement à l'origine de déplacements vers le pôle urbain voisin, elle est aussi traversée par des déplacements issus de communes plus éloignées et également à destination du pôle urbain. Faute de contournement routier, les artères centrales des communes de première couronne, qui sont aussi des voies départementales, deviennent très congestionnées (Charmes, 2011). C'est ainsi que ces communes se mettent à réclamer des voies de contournement et à souhaiter que l'on limite plus strictement encore l'étalement urbain (sans pour autant admettre qu'elles sont les premières responsables de l'étalement qu'elles déplorent). Une commune située au sudouest de Lyon, à 20 kilomètres du centre, est ainsi mobilisée pour obtenir une limitation de la croissance urbaine plus à l'ouest. En 2006, un élu local déclarait, après avoir souligné l'intensité du trafic sur l'une des routes départementales de la commune qui relie les Monts du lyonnais et le centre de Lyon :

Nous, on milite beaucoup pour avoir un SCOT unique Monts [du Lyonnais] Coteaux, ce qu'on appelle le Grand Ouest Lyonnais, parce qu'on se rend compte qu'on est complètement interdépendants. Les Mont de Lyonnais sont en train d'y réfléchir, parce 
qu'ils n'ont pas de SCOT [un SCOT des Monts Lyonnais, distinct de celui de l'Ouest lyonnais, est depuis lors en cours d'élaboration] (entretien, mars 2006).

Il est probable que les communes qui viennent d'être périurbanisées se mettront dans les décennies, voire dans les années qui viennent à pratiquer le malthusianisme de leurs aînées. La pression accrue du marché, conjuguée à la hantise de l'urbanisation, conduit très vite les maires à infléchir leur politique et à réduire fortement le rythme de croissance de la population. Ainsi, la commune d'Yzeron, dans les Monts du Lyonnais, a rapidement crû en même temps qu'elle a intégré l'aire urbaine lyonnaise. Elle est ainsi passée de 750 à 1000 habitants au milieu des années 2000. Cette croissance a été bien perçue car, selon son maire, elle permettait de «stabiliser les commerces ». Cette augmentation a entre autres été permise par la construction de quelques logements collectifs en centre-ville. Et selon le maire d'Yzeron, «nous avons renforcé et pas dénaturé le village. C'est une population locale qui s'est portée acquéreuse de ces nouveaux logements, notamment des retraités. Ils ont ainsi libéré des villas, dans lesquelles de jeunes couples se sont installés. » (Le Progrès, 7 janvier 2012). Mais le rythme de cette croissance est aujourd'hui mis en cause. Une fois le cap du millier d'habitants franchi, l'adoption d'un nouveau PLU en 2008 a été l'occasion de prendre des dispositions pour freiner l'accroissement de la population.

Que deviendront les ménages modestes dans ce contexte? Si la seconde couronne périurbaine s'engage, elle aussi, dans des politiques de croissance limitée, les ménages modestes qui souhaitent accéder à la propriété d'un pavillon autour de Lyon risquent de devoir s'éloigner encore plus. Cet éloignement a un important coût individuel et collectif. Les kilomètres supplémentaires sont parcourus pour l'essentiel en voiture compte tenu de la qualité très faible des dessertes en transports collectifs, ce qui a un important impact environnemental, tant en termes de consommation d'énergie qu'en termes d'émission de gaz à effet de serre. L'impact est aussi social. Pour un ménage dont les adultes travaillent, le surcoût d'un éloignement de $10 \mathrm{~km}$ est d'environ $200 €$ par mois pour les seuls déplacements domicile travail. Et d'après l'Union des maisons françaises, un tiers des acquéreurs ou constructeurs de maison individuelle neuve disposent d'un revenu mensuel net inférieur à $2400 €^{6}$. Il faut y insister fortement, l'enjeu de l'éloignement n'est pas simplement environnemental, il est aussi social, mettant en question le droit au transport et l'accès à l'emploi (Polacchini et Orfeuil, 1998).

$*$

La convergence d'intérêts à l'échelle de la métropole élargie autour des problématiques conjointes de la lutte contre l'étalement urbain et de la promotion de la densification est donc en trompe-l'œil. La première couronne périurbaine, notamment à l'Ouest, voit dans la modification de la législation nationale un atout lui permettant de verrouiller un modèle bâti sur le malthusianisme foncier, la cherté des prix immobiliers et la garantie conséquente d'un entre-soi des classes moyennes et moyennes supérieures (rappelons ici que les ménages les plus aisés résident en banlieue). Cette stratégie n'est guère remise en cause par le Grand Lyon, qui voit d'un œil relativement bienveillant la fermeture à l'urbanisation d'un Ouest consacré «poumon vert» de l'aire urbaine, territoire de résidence des classes moyennes à aisées tout autant qu'aire récréative permettant de « vendre » la qualité de vie lyonnaise à de nouvelles entreprises et à de nouveaux actifs.

\footnotetext{
${ }^{6}$ Communiqué de presse du 16 septembre 2011, en ligne, consulté en juillet 2012.
} 
Le boom démographique de la seconde couronne périurbaine sur la base de l'étalement urbain, en revanche, risque de peser sur le développement de Lyon. Il permet certes de répondre à l'épineuse question du logement des actifs à revenus modestes. Cet étalement de l'aire urbaine a toutefois un coût élevé pour ces actifs, dont certains manifestent d'ailleurs leur mécontentement en votant pour l'extrême droite (Charmes, 2012). Cet éloignement pèse aussi sur les dynamiques économiques métropolitaines : ces actifs sont indispensables au bon fonctionnement d'une agglomération souhaitant intégrer la «première division » des métropoles européennes les plus compétitives; ils constituent selon Ludovic Halbert (2010) une composante essentielle de l'avantage comparatif des métropoles. Par ailleurs, le développement démographique de la seconde couronne pourrait bien se doubler, à terme, de velléités de développement économique concurrençant directement le cœur métropolitain. En tout cas, pour l'instant, l'exil des actifs à revenus modestes prive le Grand Lyon de ressources fiscales.

\section{2. «Densifier pour mieux régner » : le nouveau défi des autorités métropolitaines}

Face à l'institutionnalisation de territoires périurbains captant une part croissante de ressources fiscales et démographiques et aptes à défendre leurs intérêts et leur modèle de développement axé sur la préservation de faibles densités, la modification de la forme urbaine, cette fois par la densification, s'impose à la communauté urbaine pour poursuivre, elle aussi, le développement de son territoire. Le « nouveau » modèle du cœur métropolitain est en partie la face complémentaire du modèle périurbain : le maintien de densités faibles dans les couronnes périurbaines lyonnaises et la limitation de l'étalement urbain favorisent le renouveau du cœur métropolitain par l'accroissement de la densité. Cette complémentarité est toutefois relative. C'est aussi parce qu'elle est confrontée à l'impossibilité de s'étendre sur ses marges, du moins institutionnellement, que la ville est contrainte en retour de renforcer ses propres caractéristiques, confortant par là même la division entre le cœur métropolitain et les couronnes périurbaines qui caractérise l'aire urbaine.

Par ailleurs, cette densification en partie contrainte du cœur métropolitain constitue elle-même un processus complexe : la ville ne se densifie pas partout de la même manière ni au même rythme. Il convient donc dans la seconde partie de cet article de poursuivre notre analyse géopolitique des politiques métropolitaines de compacité à une échelle plus fine, en « zoomant » sur le cœur métropolitain afin d'observer les variations de la densification selon les zones internes à l'agglomération. En effet, la volonté de la communauté urbaine se heurte à des configurations politiques, sociales et économiques communales très différenciées. À l'échelle du cœur métropolitain, la politique de densification varie donc fortement selon les profils des communes et la nature des relations entre leurs représentants et la communauté urbaine. La longueur limitée de cet article ne nous permettra pas d'analyser précisément l'échelle « micro », celle des politiques de densification mises en œuvre dans les communes. Toutefois, la ségrégation caractérisant historiquement la métropole lyonnaise entre la banlieue Est et la banlieue dite « Ouest $»^{7}$ fournit un outil d'analyse précieux pour rendre compte de la réception de l'incitation pour la densification selon le secteur du cœur métropolitain - tout en gardant à l'esprit, évidemment, que l'Ouest comme l'Est de la métropole lyonnaise constituent des territoires eux-mêmes très hétérogènes.

\footnotetext{
${ }^{7}$ Comme l'indique la carte 5, les concentrations de richesse se trouvent surtout au Nord-Ouest, mais l'usage, que nous avons repris, veut que l'on parle des banlieues Ouest.
} 


\section{1. «Densifier sans se démettre » : le Grand Lyon et la densification «conservatrice» des banlieues}

Les contraintes liées au fonctionnement du marché foncier et la saturation du centre de la métropole, constitué par les villes de Lyon et Villeurbanne, canalisent naturellement la densification vers les marges du cœur métropolitain, c'est-à-dire la banlieue lyonnaise. Or le changement de morphologie urbaine y présente dans l'ensemble des risques politiques prégnants. De nombreux travaux montrent en effet la capacité de mobilisation des habitants des banlieues résidentielles lorsque leur cadre de vie leur paraît menacé (Dziedzicki, 2003). L'attachement à la propriété d'une maison individuelle dans des quartiers de faible densité est d'autant plus fort que d'une part cette possibilité n'a été acquise que récemment pour la majorité de la population; d'autre part, des représentations négatives sont désormais associées aux quartiers de grands ensembles et à leur population. De ce point de vue, la banlieue Est de Lyon a régulièrement connu des émeutes urbaines. La «panique morale » qui s'ensuit (Wacquant, 2006) renforce l'hostilité des pavillonnaires du cœur métropolitain lyonnais à l'encontre d'une forme urbaine dense. Par ailleurs, la défense du cadre de vie se double souvent d'un attachement à une forme d'entre-soi social. Or la densification d'un territoire semble susceptible d'en modifier la composition sociale. Cette dimension sociale de la densification est d'ailleurs bien présente dans la loi SRU de 2000, l'urbanisation promue par l'État français visant désormais explicitement la mixité sociale (Epstein et Kirszbaum, 2003).

Comment mettre en œuvre une politique de compacité urbaine dans les banlieues lyonnaises tout en préservant l'équilibre social et économique de l'agglomération, marqué par une ségrégation historique entre la banlieue Ouest, plutôt résidentielle et souvent très aisée, et la banlieue Est, plutôt industrielle et populaire (voir carte 5)? Tel est l'objectif du compromis négocié au cours de la dernière décennie entre les maires des communes de banlieue et l'exécutif de la communauté urbaine dirigé par le maire de Lyon, Gérard Collomb. Ce compromis résulte avant tout d'une négociation politique entre les échelles locales de gouvernement. Il se donne à voir dès 2002, au moment où est lancée l'élaboration du SCOT de l'agglomération lyonnaise. Tout en affichant une volonté générale de mettre fin à l'étalement urbain et de densifier l'ensemble de l'agglomération lyonnaise, ce SCOT reflète la vision différente de la densification entre les banlieues Ouest et Est de la ville, traduisant le compromis entre l'intercommunalité et les communes des banlieues. Ainsi, les enjeux différenciés de la densification de l'agglomération lyonnaise marquée par une relative ségrégation socio-spatiale opposant l'Est et l'Ouest, sont clairement indiqués dans les premières orientations du SCOT en 2004 :

Au-delà des évolutions générales qu'ont connu l'agglomération et les enjeux auxquels elle doit chercher à répondre, la réalité physique et géographique du territoire oblige à effectuer un diagnostic différencié selon les secteurs. Ainsi, l'Est et le Sud, territoires d'expansion "naturelle » de l'agglomération, sont porteurs de lourds enjeux de développement; sur ces secteurs, un travail de composition et de ménagement des espaces urbanisés apparaît nécessaire. L'Ouest et le Nord, espaces plus préservés, organisés autour de collines et de vallons, doivent quant à eux mieux intégrer les logiques de développement de l'agglomération. Enfin, le secteur centre, porteur d'enjeux de développement métropolitains, doit conforter son rôle tout en s'ouvrant sur les territoires périphériques. 
La stratégie de densification affichée à l'échelle du cœur métropolitain vise apparemment à rééquilibrer la forme urbaine de l'agglomération, mais la mise en œuvre de cette densification ne bouleverse pas l'équilibre historique des banlieues lyonnaises. Les politiques appliquées localement sont relativement conservatrices. Comprendre ce traitement différencié des banlieues lyonnaises dans le partage de la charge de la densification nécessite de se pencher sur les compromis noués au sein du cœur métropolitain. Ceux-ci diffèrent grandement selon le profil économique, social et politique des communes de banlieue. Nous allons donc à présent observer successivement la négociation politique de la densification dans deux territoires de banlieue très contrastés : la banlieue Ouest, puis la banlieue Est.

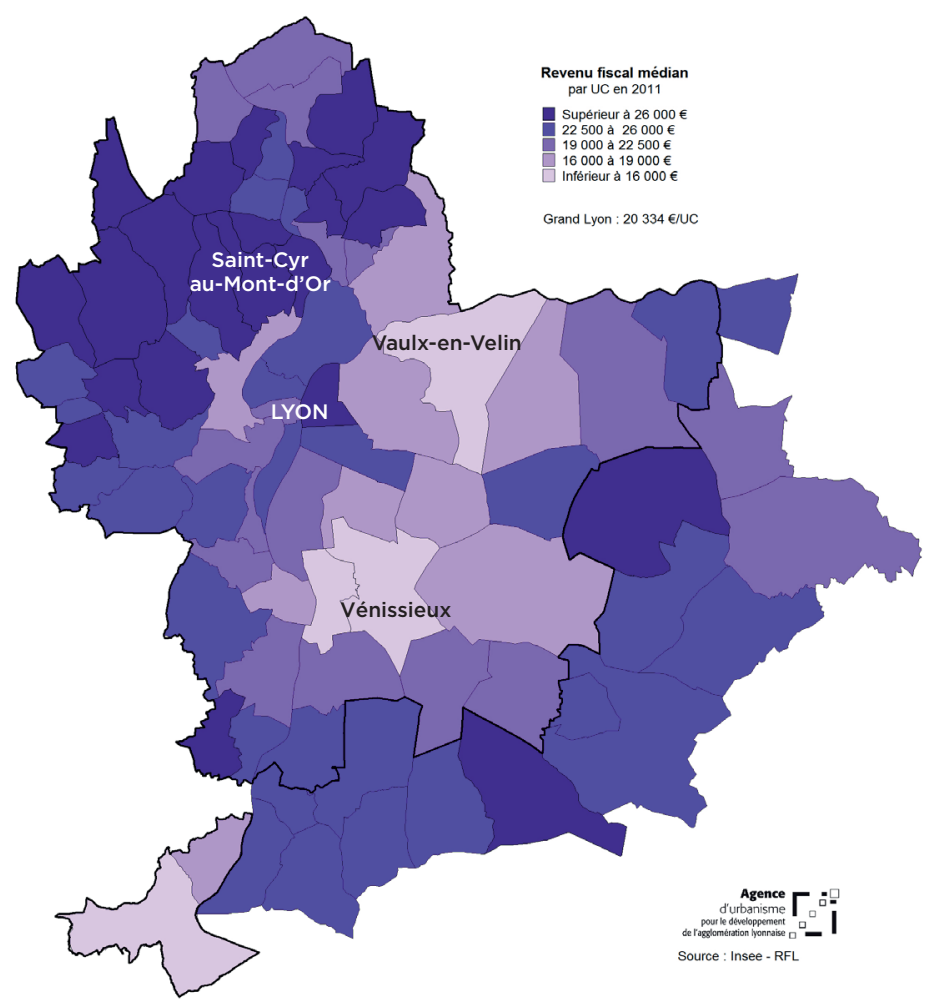

Carte 5 : Répartition des revenus dans le territoire couvert par le SCOT de l'agglomération lyonnaise. Le périmètre du Grand Lyon apparaît en traits épais (document de l'Agence d'urbanisme de Lyon).

\subsection{Banlieue Ouest : contrôler la densification afin de préserver le statu quo social}

La densification constitue davantage un risque politique pour les maires des communes de l'Ouest que pour ceux de l'Est, pour des raisons avant tout liées à la composition sociale générale des deux zones. La recherche urbaine a bien montré le lien entre la possession de capital culturel et la fréquence des mouvements NIMBY (Sénécal et al., 2002). À l'Ouest, les habitants s'organisent plus facilement avec succès pour bloquer des projets de densification même restreints, en utilisant l'image repoussoir des grands ensembles et de leurs 
habitants. Ces résistances concernent particulièrement les quartiers pavillonnaires, où il est très difficile de relâcher les contraintes réglementaires sans provoquer des réactions de défense. Dès lors, le compromis fréquemment passé entre les élus de ces communes et la communauté urbaine concerne une densification par du petit collectif dans les noyaux d'urbanisation anciens situés au cœur de ces communes. Très généralement, les élus de l'Ouest perçoivent trois grands avantages à la densification de leurs centres anciens (cœurs de bourg ou de village). Premièrement, elle répond à une véritable demande, émanant en particulier des personnes âgées de la commune désireuses de se rapprocher des services communaux. Deuxièmement, elle permet de stabiliser une armature commerciale, répondant davantage à une demande familiale. Troisièmement, elle permet d'accroître les ressources fiscales de la commune. Toutefois, outre ces arguments publics, la canalisation de la densification vers les cœurs anciens résulte également d'un compromis généralement moins affiché : elle permet de préserver en échange le statu quo pour les quartiers pavillonnaires de grandes parcelles. Comme l'indique un élu d'une commune aisée de l'Ouest lyonnais :

Toutes les majorités ont toujours été tétanisées par la croissance de la population. Il ne faut pas que la population augmente. Or le discours politique global de Collomb, c'est "il faut accueillir de nombreuses personnes chaque année dans l'agglomération. Tout le monde doit prendre une partie de sa charge ». Le compromis qu'on avait élaboré, c'était : "la commune doit se développer, OK. Alors on va développer les zones [comprendre cour] de bourg, on va favoriser cela» (entretien, élu d'une commune de la banlieue Ouest, octobre 2011).

Le compromis autour d'une politique de compacité urbaine triplement circonscrite (du petit collectif, dans le noyau ancien, dont la part de logements sociaux est strictement contrôlée) résulte d'une stratégie politique collective mise en œuvre par les maires de l'Ouest lyonnais, un territoire majoritairement gouverné par le centre-droit (voir carte 6). En 2007, un député local, qui vise alors la présidence de la fédération départementale de l'UMP du Rhône, tente de leur imposer l'étiquette UMP. Les maires de l'Ouest refusent et forment un nouveau groupement politique de centre-droit, Synergie. Comme l'indique un élu de l'opposition socialiste de l'Ouest :

[Le député UMP] leur a mis une telle pression que certains qui voulaient passer la main (...) se sont dit que leur successeur n'allait pas tenir le coup. Toute cette éclosion de Synergie signe l'échec de la stratégie de l'UMP. Ensuite Collomb a groupé les maires par secteurs et organise des débats avec les services techniques. Il les bichonne. Du coup, les maires Synergie ont fini par faire des concessions sur les logements sociaux: dès que les élections sont passées, on y va! Nous à gauche, on s'est retrouvés à devoir les appuyer à leur demande! Le contexte politique est donc différent (entretien, élu d'une commune de la banlieue Ouest, octobre 2011). 


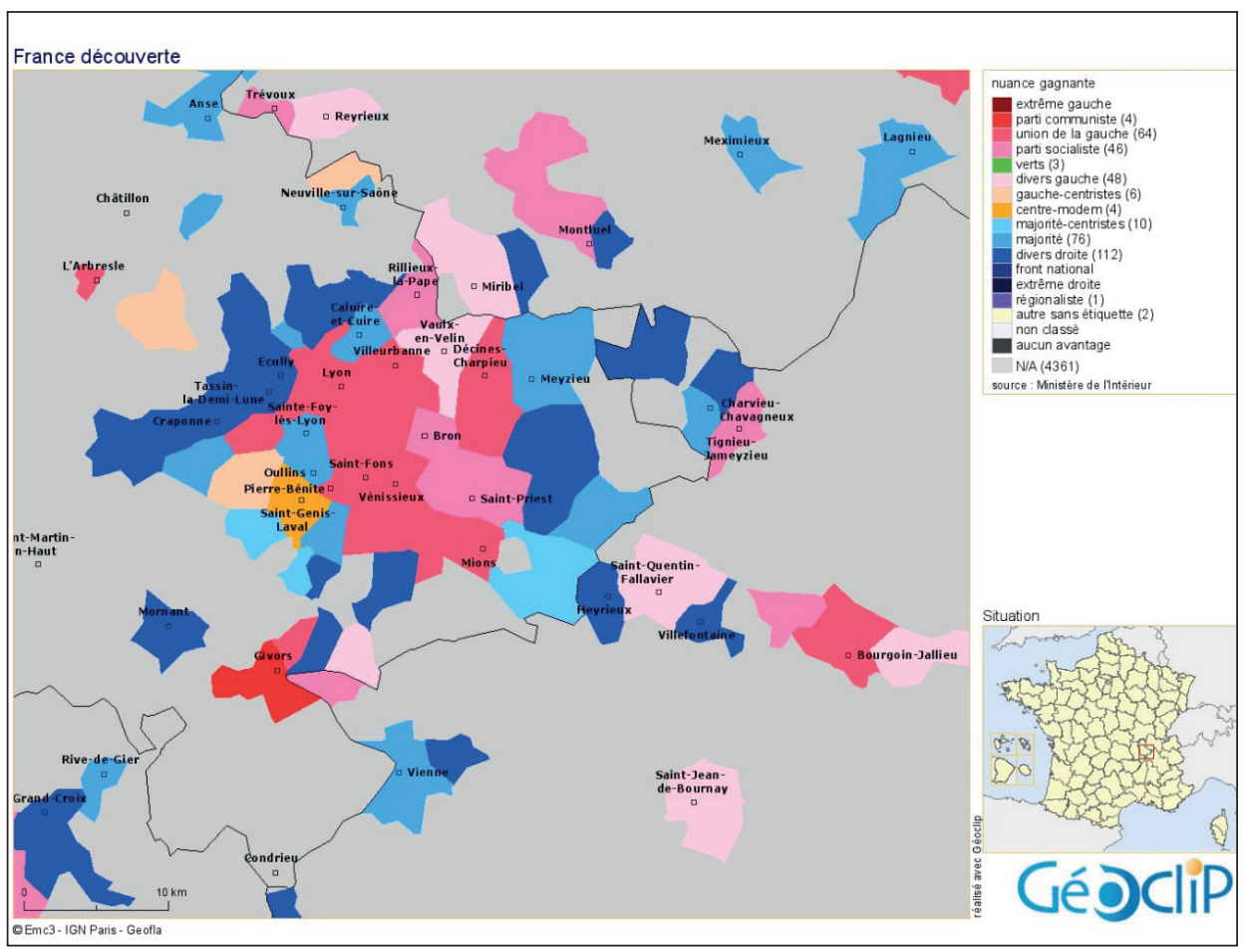

Carte 6 : Couleurs politiques des communes dans la région métropolitaine de Lyon. On note le poids des maires divers droites dans les secteurs les plus aisés de l'agglomération (source : Géoclip, 20138).

La question d'une ouverture contrôlée des communes de l'Ouest au logement social et à la densification, à laquelle l'UMP est fermement opposée, apparaît dès lors comme un terrain d'entente possible entre les élus de l'Ouest et la communauté urbaine dirigée par les socialistes, favorisant en retour l'ancrage de Synergie au détriment de l'UMP. Comme l'indique un élu de l'Ouest :

Politiquement Collomb a besoin de négocier avec l'Ouest. Il a donc passé un deal avec Synergie sur le logement social : "OK on ne fait pas des barres, on ne construit pas de logements sociaux n'importe comment mais vous en faites quand même un peu et je vous soutiens ». La communauté urbaine reconnaît que l'Ouest produit des efforts dans le logement social, en petit collectif, qui est la seule façon de passer les recours (entretien, élu d'une commune de la banlieue Ouest, octobre 2011).

La densification limitée à des petits immeubles collectifs en centre-ville, principalement dans le cadre de ZAC, permet aux municipalités de donner des gages de bonne volonté à la communauté urbaine, dont les leaders peuvent ainsi afficher que le « fardeau » de la densification s'impose indifféremment aux communes de l'Est comme de l'Ouest. Dans ce cadre, la proportion de logements sociaux s'accroît sensiblement au cours des années 2000. Ce phénomène tient pour partie à l'appui de la communauté urbaine, qui apporte les

${ }^{8} \mathrm{http}: / /$ goo.gl/zRNKGm 
fonds nécessaires à l'achat des terrains, dont le coût est nettement plus élevé dans l'Ouest que dans l'Est. Comme l'indique le maire d'une commune très huppée de l'Ouest ne comportant que $0,3 \%$ de logements sociaux : « À plus de 800 euros le mètre carré, il est clair que l'on n'aurait pas pu lancer nos programmes si le Grand Lyon ne nous avait pas aidés à hauteur de plus de 500 euros par mètre carré » (Libération, 20 décembre 2010).

Pourtant, la préservation du statu quo dans l'Ouest lyonnais n'est dans l'ensemble guère menacée par le changement de forme urbaine, car les maires ont la possibilité de contrôler non seulement la forme des constructions, mais aussi le profil des populations résidentes. Du fait de leur rôle important dans l'attribution des logements sociaux, ceux réalisés dans les nouveaux programmes permettent de loger les jeunes de la commune, dans un contexte de montée continue des prix de l'immobilier au cours des années 2000. Ces arguments sont souvent évoqués par les maires auprès de leurs administrés afin de permettre la densification a minima de la banlieue Ouest et le respect des seuils de logements sociaux imposés par la loi SRU de 2000. Comme l'indique le maire d'une commune résidentielle de l'Ouest:

Les gens imaginent des barres d'immeubles avec des antennes paraboliques. Alors, on montre les maquettes. On explique que ce sont de petites constructions, respectueuses de l'environnement patrimonial. On explique aussi que cela peut permettre aux couches moyennes et à leurs enfants de rester sur la commune (Libération, 20 décembre 2010).

Par ailleurs, de nombreux résidents des nouvelles constructions ne proviennent pas d'autres communes de l'agglomération lyonnaise, mais bien de la commune elle-même : comme dans les premières couronnes périurbaines, la densification répond aussi aux besoins d'une population âgée désireuse de se rapprocher des commerces du centre. Cette démonstration par les municipalités des communes résidentielles de l'Ouest de leur aptitude à contrôler la population amenée à résider dans les nouveaux logements constitue la condition sine qua non à la densification : même si les recours déposés par les associations de riverains aboutissent rarement, ils sont susceptibles de faire échouer les projets de construction en raison des surcoûts provoqués par les délais. Dès lors, l'étroit contrôle politique de la densification permet un compromis acceptable pour l'ensemble des parties. Ce contrôle limite les conséquences sociales du changement morphologique des communes de l'Ouest.

Bref, dans un contexte où l'incitation à densifier est perçue comme une menace par les communes de la banlieue Ouest de Lyon (celle de perdre le «cachet de village » au profit de l'avènement de véritables « villes », c'est-à-dire de passer du statut d'espaces purement résidentiels, socialement homogènes et relativement fermés, à celui d'espaces ouverts et aux fonctions diversifiées sous l'effet de l'intensification de l'urbanisation), le compromis entre la communauté urbaine et les maires de l'Ouest permet à l'ensemble des parties d'afficher des réalisations en termes de densification tout en préservant l'essentiel, c'est-à-dire un relatif statu quo urbain et social.

\subsection{Banlieue Est : promouvoir la densification au nom de la croissance}

En revanche, la densification est dans l'ensemble bien davantage appuyée par les municipalités des banlieues populaires de l'Est lyonnais. Deux raisons expliquent ce phénomène. Premièrement, la densification conduit à une demande accrue d'équipements communaux (écoles, crèches etc.). Or de nombreuses villes de l'Est ont connu une décrue démogra- 
phique. Ces communes détiennent donc déjà les équipements publics susceptibles de satisfaire les demandes d'une nouvelle population, et la densification apparaît comme une solution afin de rentabiliser ceux-ci. Ainsi, le déclin antérieur de Vaulx-en-Velin constitue l'un des supports de l'intensification actuelle, même si l'étincelle a été fournie par une politique métropolitaine d'aménagement (la construction d'une nouvelle ligne de tramway) qui a permis d'attirer promoteurs et nouvelles populations. On retrouve le même phénomène à Bron, où le maire explique : « on a les équipements pour 40000, on est redescendu à 36000 , on peut densifier. Au contraire, il faut éviter que la population continue à baisser car cela mettrait en péril les équipements de la ville conçus pour 40000 habitants. »

Secondement, la modification de la forme urbaine apparaît aux yeux de nombreux élus de ces communes comme un levier de changement social : la densification permet d'attirer des membres des classes moyennes, et d'accroître le turn-over de la population locale. L'incitation à densifier rejoint ici les préoccupations des élus des villes d'anciennes industries en déclin pour la gentrification (Rousseau, 2008). Par ailleurs, politiquement, les communes des banlieues de l'Est sont davantage gouvernées par la gauche. Les maires de ces communes appartiennent à la majorité politique gouvernant la communauté urbaine, ce qui leur offre l'accès aux ressources communautaires permettant de stimuler la densification, comme la politique de transports publics. Pour les élus et les techniciens municipaux de la banlieue Est de Lyon, le changement social résultant du changement de forme urbaine permet en premier lieu de «casser la ghettoïsation » (entretien, service d'urbanisme d'une commune de l'Est lyonnais, mars 2011) en renforçant la cohésion et le contrôle social dans des villes dont une part importante de la population est paupérisée. En second lieu, elle permet de soutenir le développement d'une armature commerciale renaissante dans les centres-villes. Profitant de l'augmentation rapide des prix immobiliers du centre de l'agglomération, des communes de l'Est lyonnais telles que Vénissieux et Vaulx-en-Velin se sont depuis peu spécialisées dans l'accueil d'une population spécifique : «des primo-accédants, pas encore avec des enfants, jeunes, majoritairement originaires de l'Est lyonnais » (entretien, service d'urbanisme d'une commune de l'Est lyonnais, avril 2011). La cible de la politique de densification n'est donc pas les habitants aisés du reste de la métropole, mais les jeunes ménages issus de l'Est en ascension sociale. Le lien établi par la législation nationale entre la densification et l'avènement de la mixité sociale est ainsi inversé : la politique de densification ne vise pas à attirer des couches populaires dans des quartiers de classes moyennes, mais bien l'inverse. Dans une commune de l'Est lyonnais longtemps sujette au déclin économique et à une décrue démographique, le retour des classes moyennes est ainsi perçu :

On part du principe que la concentration de précarité est productrice de violence et de déviance mais a contrario que la concentration de richesse n'est qu'un élément de concentration de précarité sur d'autres secteurs, d'autres territoires. La volonté à l'échelle de l'agglomération, c'est de casser cela. Cela veut dire qu'il faut rechercher sous le terme de mixité sociale l'apport de classes moyennes qui sont génératrices de besoins nouveaux et qui confortent une certaine modernité du territoire, une certaine attractivité du territoire (entretien, service d'urbanisme, février 2011).

À Vaulx-en-Velin et à Vénissieux, les espaces du renouveau urbain se démarquent ainsi nettement des grands ensembles issus de l'urbanisation fordiste qui ont longtemps régné sur les paysages urbains de l'Est lyonnais, en produisant une image négative associée au 
« communautarisme », à la pauvreté et à l'insécurité. Il s'agit cette fois, au contraire, d'une urbanisation menée par des grands groupes immobiliers privés qui reproduisent une forme urbaine dense mais dont l'esthétique est apte à attirer les classes moyennes. Vénissieux en constitue un bon exemple. Ville populaire de la première couronne de l'Est lyonnais marquée par un tissu industriel en voie de délitement, la commune se caractérise par un tissu mixte, mêlant habitat pavillonnaire et collectif ainsi que de nombreux terrains à vocation économique. Commune issue de la première vague de suburbanisation (la ville passe de 15000 habitants en 1946 à 75000 en 1975), Vénissieux a longtemps constitué une ville dont une large partie des habitants travaillait dans les usines de la commune. La fermeture de nombre de ces dernières dans les années 1970 a entraîné d'importants problèmes sociaux et la ville a vu sa population décroître rapidement (55000 habitants en 1999). Vénissieux a connu une sorte de « white flight » à la française, car ce sont avant tout les habitants qui pouvaient accéder à la propriété qui ont quitté la commune. Les tours de la ZUP des Minguettes, théâtre de plusieurs émeutes urbaines et symboles du « problème des banlieues » en France, sont aujourd'hui détruites pour laisser place à du petit collectif privilégié par la municipalité. La re-densification observée depuis le milieu des années 2000, qui a permis à la ville de regagner 7000 habitants, est promue par la municipalité communiste, qui tire profit de la politique de transport de la communauté urbaine.

Sous l'effet de l'arrivée du tramway en centre-ville et de la pression foncière croissante, on note depuis peu une mutation du tissu pavillonnaire en centre-ville : des promoteurs d'envergure nationale achètent des maisons, effectuent un remembrement foncier et construisent du petit collectif ciblant les jeunes ménages actifs travaillant à Lyon mais exclus des logements du centre de l'agglomération par la montée des prix de l'immobilier. Cette forme de gentrification est bien accueillie par la municipalité car elle permet de développer l'emploi qualifié dans la ville, mais également de développer l'armature commerciale communale afin de créer un véritable centre-ville à Vénissieux. Par ailleurs, la nouvelle attractivité de Vénissieux, qui se concrétise par le triplement des permis de construire instruits entre 2005 et 2010, accroît la capacité de négociation de la municipalité vis-à-vis des promoteurs, lui permettant de demander davantage de « qualité » et d'imposer des contraintes architecturales aux nouveaux projets afin de stabiliser la nouvelle fonction résidentielle de Vénissieux :

Les promoteurs viennent nous voir assez en amont, dès qu'ils ont une vente, ils viennent nous voir. La négociation porte sur le projet, l'implantation, le détail architectural, les espaces verts intégrés. On essaye d'accompagner des projets. Quand ils partent dans tous les sens, on leur demande de mettre un peu de cohérence. C'est au promoteur qui signera le plus vite. (...) On essaye de limiter la densification : il s'agit de ne pas faire un bétonnage au maximum. Il y a un passé sur la commune, d'où une volonté de ne pas recréer des situations où on empile les gens les uns sur les autres. On recherche la qualité de vie, aussi bien pour le privé que pour le social (entretien, direction du service de l'urbanisme de Vénissieux, mars 2011).

Notons enfin que la densification de la banlieue Est de Lyon se voit dans l'ensemble facilitée par la faiblesse de la mobilisation des habitants en comparaison de la banlieue Ouest. Il convient toutefois de préciser que des mouvements d'opposition à la densification se sont récemment structurés à l'Est. Ceux-ci proviennent avant tout des anciens noyaux villageois et des îlots pavillonnaires implantés entre les grands ensembles, comme à Vaulx- 
en-Velin où une association s'oppose actuellement avec un succès croissant aux nouvelles constructions d'immeubles (Rousseau, 2012). Au-delà même de la coupure entre les banlieues Est et Ouest, la principale opposition aux politiques de compacité urbaine provient ainsi d'un type de tissu urbain particulier: le tissu pavillonnaire. Généralement relativement âgés, les propriétaires de ces pavillons, issus plutôt de la classe moyenne supérieure à l'Ouest et davantage de la classe moyenne inférieure à l'Est, constituent clairement la principale source d'opposition aux politiques de densification du pôle urbain.

\section{Conclusion : les enjeux politiques, sociaux et économiques de la compacité}

Cet article le confirme, les politiques de compacité sont loin d'être purement techniques. L'idéal de la ville compacte peut s'incarner de manières variables, avec par exemple la densification des noyaux villageois plutôt que la transformation des quartiers pavillonnaires (Touati, 2013). Par ailleurs, les politiques de lutte contre l'étalement sont contrecarrées par d'autres, notamment celles qui soutiennent le secteur de la construction immobilière résidentielle et l'accession à la propriété. Dans ce contexte, les choix faits ont une valeur politique, économique et sociale. En termes géographiques, ces choix se distribuent inégalement en fonction des contextes locaux et ils s'articulent les uns aux autres dans une dynamique géopolitique.

Dans les couronnes périurbaines, les politiques menées au nom de la lutte contre l'étalement urbain sont diversement appropriées. Contrairement à ce qui se passe dans le cœur métropolitain, cette diversité s'organise peu à partir des appartenances politiques dans la mesure où les maires sont souvent sans étiquette (voir carte 6). Même si ce n'est évidemment pas le seul facteur à prendre en compte, les variations proviennent avant tout des positions géographiques et notamment de l'éloignement, éloignement qui se cumule avec une baisse des revenus des ménages et avec l'importance croissante de la ruralité. Dans les communes les plus proches du cœur métropolitain, les politiques de compacité entrent en résonance avec la volonté des habitants de limiter la croissance. Ces habitants peuvent imposer leur choix d'abord en raison de la fragmentation des pouvoirs communaux, avec $85 \%$ des communes périurbaines qui comptent moins de 2000 habitants. Mais ces choix sont confortés et renforcés par les mécanismes de régulation métropolitains mis en place au nom du développement durable et de l'impératif de lutte contre l'étalement urbain.

Ces politiques des communes des premières couronnes périurbaines s'ajustent doublement à celles menées dans le reste de la région métropolitaine. Premièrement, elles favorisent le report de la demande de logement sur les communes les plus éloignées du cœur métropolitain. Ces dernières, et plus particulièrement leurs propriétaires fonciers, peuvent alors tirer parti des plus-values apportées par l'urbanisation. Elles peuvent également redynamiser leur démographie et rouvrir des classes. Elles le peuvent d'autant plus facilement que, placées à l'écart de la métropole, elles subissent peu les contraintes de la lutte contre l'étalement. Secondement, le relatif blocage de la construction dans les premières couronnes périurbaines soutient la structure intercommunale de l'agglomération lyonnaise dans les projets de densification des territoires dont elle a la charge. La géopolitique métropolitaine des politiques de densification opère dès lors à une échelle plus fine, celle de l'agglomération. 
Au cœur de la métropole, les politiques de densification sont elles aussi diversement réceptionnées et varient selon les spécificités économiques, sociales et politiques des communes. C'est ainsi que les politiques de densification mises en œuvre dans l'agglomération lyonnaise n'infléchissent pas la ségrégation historique entre la banlieue Ouest, plutôt résidentielle et souvent huppée, et la banlieue Est, plutôt industrielle et populaire. Au contraire, le compromis politique noué entre plusieurs maires de l'Ouest lyonnais et la communauté urbaine permet de transformer la densification en un vecteur de renforcement de la spécialisation résidentielle de l'Ouest. De même, celui noué entre des maires de l'Est et la communauté urbaine permet de transformer la densification en un vecteur de renforcement de la spécialisation de l'Est, tournée vers le développement économique. Les jeux politiques des institutions mettant en œuvre la densification et la recherche du consensus caractérisant le fonctionnement général des intercommunalités françaises (Desage et Guéranger, 2011) ne permettent donc pas de redistribuer les fonctions et les couches sociales à l'échelle du cœur métropolitain, et de mettre ainsi fin à la division sociale des banlieues lyonnaises (Rousseau, à paraître).

À l'échelle de l'aire urbaine, les enjeux ne sont pas moins lourds. Pour la métropole lyonnaise comme pour d'autres, et notamment pour Paris, un problème lancinant est celui du déficit de production de logements. L'insistance sur la densification au nom du développement urbain durable semble de nature à réduire ce déficit. Mais comme nous l'avons observé, ce que l'idéal de ville compacte apporte à l'exigence de production de logement, il le reprend au nom de la lutte contre l'étalement urbain et de la préservation des espaces non bâtis autour des métropoles. Ces exigences favorisent un malthusianisme foncier qui réduit les droits à construire dans des secteurs où la demande de logements est pourtant très forte et où les opérateurs du marché immobilier interviendraient volontiers. Ainsi, en l'état actuel des dynamiques dans l'aire urbaine de Lyon, il est permis de douter que la production de logements sera à la hauteur des besoins. Le déficit structurel de logements risque de se maintenir et de n'être qu'en partie résorbé par une avancée de l'aire urbaine plus loin dans la région métropolitaine. Bien évidemment, ce seront les plus modestes qui en souffriront. D'ores et déjà, la dynamique du marché immobilier et la centrifugeuse périurbaine rendent difficiles et précaires la situation des accédants à la propriété modestes (les ménages dont le revenu est inférieur à 3 SMIC constituaient en 2010 plus de la moitié des accédants sur l'ensemble du territoire national [ONPES, 2012]). Pourtant, comme nous l'avons rappelé avec Ludovic Halbert (2010), le dynamisme économique des métropoles repose tout autant sur les ménages peu qualifiés à revenus modestes et sur les classes moyennes inférieures que sur les cadres supérieurs. Dans la perspective d'un développement durable de la métropole lyonnaise, les conséquences sociales, mais aussi environnementales, de l'exil progressif des actifs modestes ne sont pas anodines.

La géopolitique métropolitaine des politiques de compacité a également des conséquences économiques. Une théorie du développement territorial actuellement influente indique que la performance métropolitaine repose notamment sur un couplage réussi entre activités productives et activités « résidentielles » (Davezies, 2008). Dès lors, la compétition internationale entre les grandes métropoles ne reposerait plus seulement sur l'offre d'emplois bien rémunérés, mais sur l'offre d'un «package » couplant harmonieusement une offre d'emploi de qualité et diversifiée et un ensemble d'aménités « résidentielles », l'ensemble garantissant la croissance économique et démographique 
soutenue de la métropole. Au sein du Grand Lyon, les politiques de densification restent indéniablement susceptibles de contribuer à limiter les effets de la saturation générale du marché immobilier à l'échelle de la métropole lyonnaise. Et de fait, $80 \%$ des ménages venus s'installer dans l'aire urbaine de Lyon entre 2002 et 2007 en provenance du reste de la France se sont installés dans le périmètre du Grand Lyon.

Ceci étant dit, ces chiffres sont établis sur la base du périmètre de l'aire urbaine défini en 1999 et l'aire urbaine de 2010 est beaucoup plus large : calculé sur la base de ce dernier périmètre, le taux de captation des nouveaux ménages par le Grand Lyon serait beaucoup plus faible. Plus généralement, les atouts du périurbain lyonnais semblent loin d'être étrangers à l'attractivité d'ensemble du territoire (Chabanel, 2013). Dans cette perspective, le périurbain, encore souvent considéré comme le «parasite fiscal» des cœurs métropolitains, pourrait apparaître de manière plus positive comme un « levier de captation de revenus résidentiels » (tirés des actifs locaux comme des touristes), tant ses faibles densités offrent un cadre privilégié aux activités récréatives (Davezies, 2013); toutefois, ceci nécessiterait de parvenir, sinon à une intégration politique de l'aire urbaine, du moins à une véritable coopération entre l'agglomération et ses couronnes périurbaines. À première vue, les bases de cette coopération existent. Mais la convergence d'intérêts est en réalité en trompe-l'œil. L'actuel équilibre géopolitique entre le cœur métropolitain et ses hinterlands périurbains semble de nature à empêcher la mise en place d'une stratégie de développement intégrée de l'aire urbaine et contrecarre ainsi très clairement les aspirations actuelles de la communauté urbaine à inviter Lyon à la table des «city-regions » parmi les plus compétitives d'Europe. 


\section{Bibliographie}

Beal V., Gauthier M., Pinson G., 2011. Le développement durable changera-t-il la ville? Le regard des sciences sociales. Presses de 1'Université de Saint-Étienne, Saint-Étienne.

Cavailhès J., 2004. L'Extension des villes et la périurbanisation In Institut des villes, Villes et Économie, La Documentation française, Paris, 157-184.

Chabanel, B., 2013. Le ressort de la captation de revenus. Consolider l'attractivité résidentielle et touristique de la métropole. Nova 7/Grand Lyon, Millénaire 3.

Charmes E., 2005. La vie périurbaine face à la menace des gated communities. L'Harmattan, Paris.

Charmes E., 2011. La ville émiettée. Essai sur la clubbisation de la vie urbaine. Presses Universitaires de France, Paris.

Charmes E., 2012. La vie périurbaine favorise-t-elle le vote Front national? Études foncières 156, 8-10.

Charmes E. et Keil R., à paraître, The Politics of Post-Suburban Densification in Canada and France. International Journal of Urban and Regional Research.

Crouch C., 2005. Post-Democracy. Polity Press, Cambridge.

Davezies L. et Estèbe P., 2007. Les SCOT de l'aire métropolitaine de Lyon Saint-Étienne : pôles productifs et dynamiques résidentielles. Acadie, Agence d'urbanisme de Lyon, Lyon.

Davezies L., 2008. La République et ses territoires : la circulation invisible des richesses. Le Seuil, Paris.

Davezies L., 2013. Pour une nouvelle synergie entre les grandes villes et leur hinterland. Interview réalisée par B. Chabanel, Millénaire 3.

Desage F. et Guéranger D., 2011. La politique confisquée. Sociologie des réformes et des institutions intercommunales. Éditions du Croquant, Paris.

Dziedzicki J-M. 2003. Au-delà du NIMBY : le conflit d'aménagement, expression de multiples revendications In Melé P., Larrue C. et Rosemberg M. (coord.), Conflits et Territoires, Presses Universitaires FrançoisRabelais, Tours, 35-64.

Echenique M.H. et al., 2012. Growing Cities Sustainably. Journal of American Planning Association 78 (2), 121-137.

Epstein R. et Kirszbaum T., 2003. L'enjeu de la mixité sociale dans les politiques urbaines. Regards sur l'actualité 292, 63-73.

Estèbe P., 2008. Gouverner la ville mobile. Presses universitaires de France, Paris.

Floch J.-M. et Lévy D., 2011. Le nouveau zonage en aires urbaines de 2010 - Poursuite de la périurbanisation et croissance des grandes aires urbaines. Insee première 1375.

Halbert L., 2010. L'Avantage métropolitain. Presses Universitaires de France, Paris.

Hicaubé H., 2012. Le traitement de l'étalement urbain dans la coopération interterritoriale, L'Inter-Scot de l'aire métropolitaine lyonnaise, travail de fin d'études sous la direction d'E. Charmes, Vaulx-en-Velin, ENTPE.

Jouve B., 2001. Lyon recherche gouvernance urbaine désespérément. Cahiers de géographie du Québec 45 (125), 287-292.

Levesque R., Liorit D. et Pathier G., 2011. Les marchés fonciers ruraux régionaux entre dynamiques des exploitations agricoles et logiques urbaines. Économie et statistique 444-445, 75-98.

Nessi H., 2012. Influence du contexte urbain et du rapport au cadre de vie sur la mobilité en Ile-de-France et à Rome. Thèse de doctorat sous la direction d'O. Coutard, Université de Marne-la-Vallée.

Observatoire National de la Pauvreté et de 1'Exclusion Sociale, 2012. La lettre de l'ONPES 3.

OCDE, 2012. Compact City Policies, a Comparative Assessment. OECD, Paris.

Pinson G., Rousseau M., 2011. Les systèmes métropolitains intégrés. État des lieux et problématiques. Territoires 2040 3, 28-48.

Polacchini A. et Orfeuil J-P., 1998. Les dépenses pour le logement et pour les transports des ménages franciliens. Recherche Transport Sécurité 63, 31-46. 
Reigner H., 2013. Sous les pavés de la qualité urbaine. Gouvernement des territoires, gouvernement des conduites et formes renouvelées de la domination dans la ville néohygiéniste. Mémoire d'habilitation à diriger des recherches, Institut d'études politiques de Paris.

Renard V., 2006. Le Financement de la ville. Revue d'économie financière 86, 147-156.

Renard V., 2008. La ville saisie par la finance. Le débat 148, 106-117.

Rousseau, M., 2009. Re-imaging the City Centre for the Middle Classes: Regeneration, Gentrification and Symbolic Policies in "Loser Cities". International Journal of Urban and Regional Research 33 (3), 770-788.

Rousseau, M., 2012. Public Mobility as the Defining Feature of the French Post-industrial City. Theory, Culture \& Society 29 (6), 125-145.

Rousseau, M., 2014. "Many Rivers to Cross". Suburban Densification and the Social Status Quo in the Greater Lyon. International Journal of Urban and Regional Research. À paraître.

Sénécal G., Germain A., Benard J., 2002. Portrait des pratiques communautaires et locales en revitalisation urbaine et sociale sur le territoire de l'île de Montréal. INRS Urbanisation, culture et société, Montréal.

Subra P., 2007. Géopolitique de l'aménagement du territoire. Armand Colin, Paris.

Touati A., 2013. Économie Politique de la densification des espaces à dominante pavillonnaire : l'avènement de stratégies post-suburbaines différenciées. Thèse de doctorat sous la direction d'O. Coutard et J. Rutherford, Université Paris-Est, École Nationale des Ponts et Chaussées.

Vanier M., 2008. Le Pouvoir des territoires. Essai sur l'interterritorialité. Economica et Anthropos, Paris.

Vilmin T., 2006. Le Système local de l'urbanisation en maisons individuelles, étude de cas en Ile-de-France. Logiville, rapport pour le PUCA, ministère de l'Equipement.

Wacquant L., 2006. Parias urbains. Ghetto, banlieues, État. La Découverte, Paris.

Wiel M. (Coord.), 2002. Les Raisons institutionnelles de la périurbanisation. Rapport pour la DRAST et le PREDIT, Paris. 
